\title{
Doença de Chagas em Lassance, MG. Reavaliação clínico-epidemiológica 90 anos após a descoberta de Carlos Chagas
}

\author{
Chagas' disease in Lassance, Minas Gerais State. Clinical-epidemiological \\ re-evaluation ninety years after the discovery by Carlos Chagas \\ João Carlos Pinto Dias', Evandro M.M. Machado', Érika Carime Borges', \\ Eliana Furtado Moreira ${ }^{2}$, Claudia Gontijo ${ }^{3}$ e \\ Bernardino Vaz de Mello Azeredo ${ }^{4}$
}

\begin{abstract}
Resumo Analisa-se a trajetória da doença de Chagas em Lassance (município da descoberta de Carlos Chagas) entre 1.908 a 2.001, através de registros históricos e pesquisas atuais. O município foi importante foco da tripanossomíase entre Chagas e os anos 1.980, mercê de infestação significativa das casas por Panstrongylus megistus e, mais tarde, Triatoma infestans, espécies que foram eficazmente controladas, nos últimos 20 anos. Importante no passado, a infecção chagásica é hoje residual, com uma prevalência geral de $5,03 \%$ e afetando basicamente os grupos etários elevados, não se encontrando soropositivos abaixo dos 20 anos de idade. O perfil clínico-epidemiológico dos chagásicos detectados é o habitual de áreas com transmissão interrompida, com a maioria dos casos em formas cardíacas benignas ou na forma crônica indeterminada, havendo ainda indicativos de formas digestivas, sendo a mortalidade ainda significativa, em grupos etários elevados. O município apresenta-se infestado por T. sordida, em baixas densidades e grande dispersão, não infectado por T. cruzi e restrito ao peridomicílio. Conclui-se que Lassance está hoje livre da transmissão da doença, devendo manter-se sob vigilância epidemiológica frente aos triatomíneos nativos no município e garantirse a atenção médica às pessoas infectadas no passado.
\end{abstract}

Palavras-chaves: Doença de Chagas. Lassance. Epidemiologia. Clínica. Controle. História.

Abstract The history and present situation of Chagas' disease in Lassance (the county where Carlos Chagas discovered American trypanosomiasis) were studied through a historical analysis and clinical and epidemiological research performed from 1999 to 2001. Lassance was an important focus of Chagas' disease from Carlos Chagas up until the 1980's, because of intensive infestation in dwellings by Panstrongylus megistus and Triatoma infestans, two important species which were efficiently controlled in the last twenty years. Human Chagas' disease was important in the past but today is only residual, affecting basically the more elderly age groups. The general prevalence is about $5.03 \%$ and no infected individuals are found below 20 years of age. The clinical and epidemiologic profile of the seropositive individuals studied is that expected in areas with interrupted transmission, most of these presenting the indeterminate or benign cardiac form of chronic Chagas'disease. Some cases of digestive Chagas' disease also seem to exist. Mortality due to the disease is still significant, affecting chiefly older age groups. The municipality still remains infested by Triatoma sordida, in low densities and high dispersion, non infected by T. cruzi and restricted to peridomestic foci. In conclusion, Lassance is now free of Chagas' disease transmission and must improve medical attention for the remaining infected individuals, as well as to maintain a permanent epidemiological surveillance against native Triatominae.

Key-words: Chagas'disease. Lassance. Epidemiology. Clinic. Control. History.

1. Centro de Pesquisas René Rachou da Fundação Oswaldo Cruz. 2. Fundação Ezequiel Dias. 3. Fundação Nacional de Saúde do Ministério da Saúde. 4. Secretaria de Estado da Saúde de Minas Gerais, Belo Horizonte, MG.

Suporte Parcial: FAPEMIG e FUNASA.

Endereço para correspondência: Dr. João Carlos Pinto Dias. Centro de Pesquisas René Rachou/FIOCRUZ. Av. Augusto de Lima 1715, $30190-002$ Belo Horizonte, MG, Brasil.

Telefax: 5531 3295-3115

e-mail:jcpdias@cpqrr.fiocruz.br

Recebido para publicação em 10/10/2001. 
"Assistimos o início de pequeno povoado e nessa época seria impossível, nas casas recém-construídas, encontrar um único hematophago transmissor. Decorridos dous annos, Lassance tornou-se um fóco intenso da molestia, sendo hoje naquelle logar, rarissimas as casas pobres livres da infestação pelo insecto"(Chagas 1911) ${ }^{5}$.

Passados 90 anos da gloriosa descoberta de Carlos Chagas, Lassance volta a importar como marco histórico e referência científica. Ali chegara Chagas, em 1907, convocado a controlar a malária que impedia o progresso da Estrada de Ferro Central do Brasil rumo a Pirapora, projeto nacional que objetivava ligar ao Rio de Janeiro todo o centro e o nordeste do Brasil. Em 1847, praticamente desabitado, o lugar consistia de terras devolutas pertecentes ao arraial de Nossa Senhora do Bom Sucesso e Almas do Rio das Velhas, sem fazendas ou assentamentos. A região era cortada por tropeiros, ligando lugares distantes como Montes Claros, Sabarabussu (Sabará), Diamantina e Coração de Jesus. Um tropeiro, Liberato Nunes de Azevedo, construiu, aproximadamente em 1850 , os primeiros ranchos às margens do Córrego Maria Grande, hoje São Gonçalo. Em 1907, surgem as primeiras fazendas dedicadas à agropecuária e à extração de látex em seringueiras e com a chegada da Estrada de Ferro, o desenvolvimento local foi impulsionado ${ }^{37}$. O primitivo arraial era muito modesto, situando-se à beira do Rio das Velhas. A alcunha Lassance foi dada em fevereiro de 1908, em homenagem ao engenheiro chefe da construção, Dr. Ernesto Antônio Lassance Cunha. O município emancipou-se em dezembro de $1953^{3037}$. À época de Chagas, Lassance era tão isolada como toda a região do Noroeste Mineiro, deprimido por grandes distâncias, pelo clima quente e por terras áridas, mas também pelo descaso programado da metrópole portuguesa, na fase colonial, e pelo império, durante toda sua vigência ${ }^{20}$. Ali as cidades eram muito esparsas: apenas Curvelo sendo assinalada nas imediações da Serra do Bicudo, área do encontro dos Rios S. Francisco e das Velhas ${ }^{24}$. De modo geral, Lassance passou por períodos de crescimento nas três primeiras décadas do século $X X$, mas, aos poucos, a região foi sendo esquecida ${ }^{37}$. Lassance hoje conta com uma população de 6.301 habitantes e tem como atividades econômicas básicas a extração de quartzo e de sempre-vivas, ao lado do cultivo de mandioca, milho, fumo e arroz. Reflorestamento com Eucalyptus, produção de carvão vegetal e pecuária de corte são outras atividades atuais. Mais recentemente, Lassance sofreu muito com a desativação do engenho de açúcar e a progressiva redução de atividades da Rede Ferroviária Federal ${ }^{2030}$.
No início, o grande problema de saúde foi a malária, também ocorrendo bócio endêmico, cretinismo e múltiplas ênteroparasitoses ${ }^{4}{ }^{14}$. O início da doença de Chagas em Lassance explode epidemicamente com a chegada da ferrovia, segundo o próprio Chagas, detectando o descobridor 29 casos agudos na área e incontáveis casos crônicos. O lugarejo foi rapidamente infestado por triatomíneos pouco antes da descoberta da doença, resultando grande pressão de transmissão do Trypanosoma cruzi à população já existente e à que chegava à época, com o crescimento do lugar. A espécie estudada por Chagas foi inicialmente o Panstrongylus megistus, principal triatomíneo intradomiciliar, ocorrente nas casas pobres e altamente infectado pelo T. cruzi ${ }^{21}$. No entanto, Chagas já noticia o Triatoma sordida e o $P$. geniculatus na região, este último vinculado à tripanosomíase do tatu ${ }^{5}$. Ficará sempre uma dúvida sobre o P. megistus em Lassance: teria sido uma espécie introduzida pelas migrações e pela Estrada de Ferro (hipótese de Chagas) ou já ali existia em ecótopos naturais e invadiu progressivamente as casas? A lógica e os fatos posteriores apontam mais para a primeira assertiva. Já o Triatoma infestans, principal transmissor da endemia chagásica no Brasil Meridional, somente irá aparecer na região muitas décadas depois de Chagas, nada tendo a ver com a esquizotripanose dos primeiros tempos de Lassance.

A doença de Chagas humana foi descoberta em Lassance e ali vem se constituindo em importante agravo até o presente. $\mathrm{Na}$ falta de grandes ações antrópicas e de políticas eficazes de saneamento e habitação, sua prevalência foi crescente, até que, nos anos 80, o programa ministerial contra a doença de Chagas foi priorizado e reverteu - praticamente em todo - País -esta tendência de expansão. O presente trabalho teve como objetivos centrais verificar a situação atual da doença de Chagas em Lassance e registrar a história da doença e de seu controle na Região. Além da parte histórica, levantada de registros e trabalhos clássicos, os principais dados da situação atual foram levantados por pesquisa direta na área, envolvendo toda a população, autoridades e uma série de instituições.

\section{MATERIAL E MÉTODOS}

A parte histórica foi tirada de documentos disponíveis, especialmente na Literatura sobre doença de Chagas. Aqui pontificam, naturalmente, os registros de Carlos Chagas e alguns outros, como de Chagas Filho e de Eurico Villela, além de importantes documentos históricos ${ }^{345720303738}$. Também, lançou-se mão de entrevistas em Lassance e com cientistas que se dedicaram ao assunto. A literatura pesquisada e registros da SUCAM/FNS forneceram dados importantes sobre o triatomismo e a prevalência da esquizotripanose na região. Os dados atuais corresponderam basicamente à doença de Chagas, buscando elementos de cotejamento com o panorama vivido por Chagas, há 90 anos. Pesquisou-se no município a infestação triatomínica, por agentes da FNS/ MG, em 1999, segundo metodologia própria para estes levantamentos ${ }^{18}$ As capturas fizeram-se pela técnica habitual de pesquisa direta, sem o uso de desalojantes químicos, sendo os triatomíneos catalogados em laboratório da FNS segundo a espécie, o estádio 
evolutivo e a infeção natural pelo T. cruzi. O inquérito sorológico também foi realizado em 1999 pela FNS/MG, na sede municipal, em sua periferia e em localidades rurais, após ampla articulação com Autoridades locais e com a população, trabalho antecedido por uma Feira de Ciências que teve como motivos principais a doença de Chagas e sua descoberta. A coleta deu-se na população geral não selecionada, através de papel de filtro padronizado (Whatmann $n^{\circ} 4$ ) e armazenada em geladeira comum, para processamento no Laboratório de Sorologia e Parasitologia da FUNED, Belo Horizonte, até 60 dias após a coleta. A técnica utilizada foi a de Imunofluorescência Indireta, com antígeno de T. cruzi elaborado por Bio-Manguinhos, tendo como cut-off a diluição de 1:40. O estudo confirmatório dos casos positivos fez-se no mesmo Laboratório, em soro colhido por punção venosa pela FNS/MG, pelas técnicas de ELISA e Imunofluorescência. A avaliação clínica fez-se por trabalho direto na cidade, em fevereiro de 2001, correspondendo à reconfirmação sorológica e exame clínico-eletrocardiográfico. Não sendo possível, no momento, a realização de $\mathrm{RX}$ de esôfago e cólon, valorizou-se simplesmente a cuidadosa anamnese sobre disfagia importante e obstipação freqüente e maior que sete dias, como indicativos de formas digestivas da doença de Chagas. Fez-se ainda uma tentativa de isolar o Trypanosoma cruzi dos pacientes chagásicos de Lassance, através de xenodiagnóstico com quarenta ninfas de Triatoma infestans em 14 pacientes examinados, conforme a descrição de Schenone et $\mathbf{a}^{32}$. Para o estudo da mortalidade, dados secundários foram levantados no SIM/DATASUS (via Internet) e no Cartório de Registro Civil da cidade, objetivando-se aquilatar o impacto atual e recente da doença. No plano estatístico, quando necessárias, as avaliações se fizeram pelo quiquadrado $\left(\mathrm{x}^{2}\right)$, considerando-se significativos os valores de $p$ iguais ou menores que 0,05.

Como pressupostos éticos, a sorologia foi antecedida por minuciosa explicação e realizada com a aquiescência das Autoridades de Saúde de Lassance (Secretário Municipal e Conselho de Saúde) e das pessoas examinadas ou seus responsáveis. Os resultados foram devolvidos à comunidade em 2001, conforme combinado, sendo os indivíduos soropositivos encaminhados à atenção médica pertinente. No tocante à pesquisa entomológica, as unidades domiciliares com pesquisa positiva no intradomicílio foram rociadas com inseticida piretróide. De modo geral, esta pesquisa serviu para reativar a vigilância entomológica em Lassance, de forma horizontalizada e com ampla participação comunitária.

\section{RESULTADOS}

Neste segmento serão basicamente apresentados os levantamentos históricos para a endemia na região, vindo a seguir os resultados atuais da pesquisa triatomínica e da sorologia para doença de Chagas em Lassance, finalizando-se com as informações clínicas e sobre mortalidade, obtidas na fase final da pesquisa.

Panorama histórico. Situação entomológica: Chagas descobriu a doença a partir da descrição do $T$. cruzi que evidenciou em $P$. megistus, capturados em habitações de Lassance, no segundo semestre de 1908. Ao que informa Chagas em 1911, esta espécie invadiu rapidamente os domicílios locais entre 1907 (época em que chegou a Lassance) e 1909, quando a infestação já era maciça. Triatoma sordida e Panstrongylus geniculatus foram encontrados no ambiente natural, associados a aves e a tatus ${ }^{5622}$. Outras espécies como Psammolestes coreodes (tertius) e provavelmente Panstrongylus diasi e Rhodnius neglectus já existiam em focos silvestres da região, tendo sido detectadas mais tarde pela SUCAM ${ }^{1936}$. Na região foi registrada, ainda, a presença esporádica e fugaz de outros triatomíneos como Triatoma brasiliensis (certamente trazido por migrantes nordestinos) e Triatoma vitticeps (provavelmente a partir de migrantes do Vale do Jequitinhonha), espécies que hoje não são mais ali detectadas $^{1927}$. Já Triatoma infestans, o mais domiciliar dos triatomíneos meridionais, não existia à época de Chagas e só chegou a Lassance após 195078111722 2836 . Essa espécie teve a maior importância em Minas Gerais, onde foi detectada a partir de 1910, originada de sua expansão em São Paulo no século anterior, e a seguir dispersando-se, progressivamente para o Norte, para finalmente atingir o sul do Piauí em 198021 343536 . Num inquérito estadual, em 1940, Martins et al detectaram em Lassance 19 exemplares de $P$. megistus (16 positivos para T. cruzi), 15 de T. sordida (todos negativos) e um de Rhodnius sp (negativo, provavelmente tratando-se de $R$. neglectus ${ }^{23}$. O T. infestans foi eliminado da área rapidamente após a priorização das atividades de controle químico pela SUCAM em 1983, já não sendo detectado na região desde o final dos anos 80 . O mesmo parece ter ocorrido com P. megistus, praticamente não mais capturado na área após 1985, o que reforça a impressão de haver ali sido exclusivamente domiciliar, introduzido justamente à época de Chagas mediante transporte passivo por populações imigradas do sull 11171928 . As taxas de infecção natural dessas espécies sempre foram mais altas para $P$. megistus e T.infestans, justamente as mais domiciliares na área de Lassance. Fica patente que essas duas espécies foram as responsáveis provavelmente exclusivas - pela doença de Chagas humana em toda a região, contribuindo mais $P$. megistus, até o período 1950-1960, seguindo-se fugazmente T. infestans até o início dos anos 80, época em que a desinsetização sistemática drasticamente eliminou os triatomíneos domiciliados em todo o País ${ }^{16}$ ${ }^{19} 36$. A partir dos anos 90, apenas detectaram-se em Lassance focos basicamente peridomiciliares de $T$. sordida e resíduos estritamente silvestres de espécies nativas como $P$. diasi, $P$. geniculatus e $R$. neglectus. São infestações praticamente restritas ao peridomicílio, bastante dispersas na área, em geral apresentando os 
triatomíneos capturados nula ou insignificante taxa de infecção natural pelo T. cruzi, o que confere à área mínimas condições de transmissão vetorial da doença de Chagas ao homem ${ }^{16} 1927$.

Doença de Chagas humana: à época de Chagas, os registros existentes são dele mesmo, de maneira esparsa e em várias oportunidades. Em célebre viagem a vários estados, em 1913, Neiva e Penna se referem claramente à forma cardíaca da esquizotripanose em pacientes examinados e assinalam significativa presença do mal de engasgo, no entanto sem relacionálo à etiologia chagásica ${ }^{26}$. Em 1916, revendo seu material, Chagas registra 29 casos agudos, mas sempre refere que estes estão em franca desproporção com centenas ou mais de casos crônicos detectados na área. Note-se que após 1916 não mais serão registrados casos agudos em Lassance, provavelmente porque nem Chagas nem outros pesquisadores irão permanecer por maiores períodos na área ${ }^{678914}$. Ao longo do tempo, na região haverá transmissão ativa da esquizotripanose humana, a julgar pelos inquéritos sorológicos a seguir sumariados:

- 1958-1960: prevalência detectada entre escolares de Lassance (10-15 anos de idade) entre 5,1 e $10 \%$, pela reação de RFC, sendo de $6,2 \%$ a média para Minas Gerais, indicando transmissão ativa ${ }^{31}$;

- 1978-1980: prevalência na população rural geral de Lassance $=37,1 \%$, sendo 88 soros positivos pela técnica de imunofluorescência entre 235 examinados. Desses 88 soros positivos, 33 (37,5\%) eram autóctones e os demais da região. Dentre os positivos, a proporção de pessoas até a idade de 6 anos foi de $9,1 \%$ e de 7 a 14 anos de $15,9 \%$, o que indicava também transmissão ativa. Para a região em torno de Lassance, as prevalências na população geral rural nesse inquérito foram: Montes Claros = 23,6\%; Sete Lagoas = 20,3\% e Patos de Minas = 23, $1 \%{ }^{17} 19$.

Registra-se que nunca se realizaram transfusões de sangue em Lassance, e que eventuais e raras transfusões praticadas sobre pacientes desse município ocorreram via de regra em Belo Horizonte, Pirapora, Curvelo ou Sete Lagoas. Assim, o mecanismo fundamental de transmissão da doença de Chagas em Lassance foi o vetorial, principalmente no período 19091980, ligado a infestações domiciliares importantes por P. megistus (até 1955-1960) e T. infestans (1960-1980). Naturalmente, houve casos de transmissão congênita, já assinalados por Chagas, mas em proporções muito menores que a via vetorial ${ }^{5}$. Não há maiores informações sobre a morbi-mortalidade da doença na área, fora as Chagas e a citação de Neiva e Penna, referentes às formas cardíacas e à ocorrência de mal de engasgo $0^{725}$. Nos casos de Chagas, a infecção aguda restringiu-se a crianças, com uma mortalidade geral de $37,9 \%$. Casos agudos não serão mais registrados em Lassance, mas eles ocorrerão em toda a região, principalmente devido à transmissão vetorial.
Assim, Cançado registrará que entre 1972 e 1985 terá detectado 29 (72,5\%) casos agudos do centro-norte de Minas duma série de 40 casos, em total maioria devido à contaminação pelo vetor ${ }^{2}$. Priorizado o controle vetorial da doença de Chagas em 1983, drasticamente irão desaparecer os casos agudos da moléstia em todo o Estado ${ }^{217}$. Quanto à fase crônica, Chagas refere sua grande freqüência na área, anotando a presença de formas clínicas indeterminada, cardíaca, digestiva e nervosa. A mortalidade à época era importante e precoce entre os indivíduos com a forma crônica cardíaca, referindo-se mau prognóstico entre alguns pacientes crônicos com exacerbações do quadro agudo $^{7}$. Avaliações regionais irão demonstrar a grande importância da doença de Chagas como agravo social até os anos 80 e 90, por conta da prevalência e do grau de morbidade detectados entre a população geral. Assim, considerando as taxas de aposentadoria homologadas pelo FUNRURAL e devidas à doença de Chagas entre 1976 e 80 , registraram-se na região taxas entre 32,3\% e 23,8\%, frente a 10,7\% para Minas Gerais. Já para a mortalidade devida à doença de Chagas, os coeficientes regionais por 100.000 habitantes ficaram entre 11,1 (sub-região de Montes Claros) e 39,9 (Sete Lagoas), contra 13,6 para Minas Gerais ${ }^{17}$. Já os livros do cartório local registram óbitos por doença de Chagas entre os moradores de Lassance desde a década de 50 até hoje.

Aspectos atuais. Situação entomológica: foram examinadas as 67 localidades rurais do Município e o centro da cidade. No total, revistaram-se 2.521 $(92,39 \%)$, unidades domiciliares das 2.729 existentes, números esses totalmente satisfatórios e significativos para a determinar a situação triatomínica. Capturaramse 539 triatomíneos, sendo Triatoma sordida a principal espécie detectada, com 526 (97,04\%) exemplares. Outras espécies foram 9 (2,05\%) exemplares de Rhodnius neglectus, 3 de Panstrongylus geniculatus e 1 exemplar de Panstrongylus megistus. Todos os insetos foram negativos para T. cruzi. Na zona urbana de Lassance foram examinadas 1.023 unidades domiciliares, encontrando-se apenas 1 peridomicílio infestado por T. sordida, exemplar adulto, negativo para T. cruzi (índices de infestação intradomiciliar de 0,00 e peridomiciliar de 0,1\%). As Tabelas 1, 2 e 3 sumariam os principais resultados da pesquisa entomológica, conforme os indicadores usuais da FUNASA ${ }^{1819}$.

O coeficiente de colonização intradomiciliar (\% de intradomicílios com presença de ninfas) em Lassance foi de $0,32 \%$ (oito intradomicílios com ninfas, sendo sete colonizações por T. sordida e duas por R. neglectus).

Infecção humana: ao todo foram colhidas amostras de soro de 716 pessoas, o que corresponde a mais de $10 \%$ do total da população do Município. Além da sede municipal, 18 localidades periféricas ou rurais foram contempladas, conforme a Tabela 4, onde os resultados globais por localidade estão apresentados. Nesta tabela 
Tabela 1 - Índices de dispersão e infestação triatomínica do Município de Lassance, MG, em 1999, por todas as espécies detectadas.

\begin{tabular}{lccc}
\hline Elemento geográfico & Examinados & Infestados & Dispersão/infestação (\%) \\
\hline Localidades & 67 & 47 & $70,15^{*}$ \\
Unidades domiciliares & 2.521 & 142 & $5,63^{\star *}$ \\
Casas & 2.521 & 16 & $0,63^{* *}$ \\
Anexos & 2.964 & 150 & $5,06^{\star *}$ \\
\hline
\end{tabular}

* dispersão; ** infestação

Tabela 2 - Infestação triatomínica por local e densidade em 142 unidades domiciliares de Lassance, MG, positivas para Triatoma sordida, em 1999.

\begin{tabular}{lccccr}
\hline & & & & \multicolumn{2}{c}{ Densidade por locais } \\
\cline { 5 - 6 } Local & Examinados & Infestados & Capturados $\left(\mathrm{n}^{\circ}\right)$ & só os infestados & todos \\
\hline Intradomicílio & 2.521 & 13 & 56 & 4,31 & 0,02 \\
Peridomicílio & 2.964 & 147 & 446 & 3,03 & 0,15 \\
\hline
\end{tabular}

Tabela 3 - Distribuição de triatomíneos capturados no intra e peridomicílio de Lassance, MG, segundo o estádio evolutivo.

\begin{tabular}{|c|c|c|c|c|c|c|}
\hline \multirow[b]{2}{*}{ Triatomíneos } & \multicolumn{2}{|c|}{ Intradomicílio } & \multicolumn{2}{|c|}{ Peridomicílio } & \multicolumn{2}{|c|}{ Total } \\
\hline & $\mathrm{n}^{\circ}$ & $\%$ & $n^{\circ}$ & $\%$ & $\mathrm{n}^{\circ}$ & $\%$ \\
\hline Adultos & 41 & 51,25 & 15 & 3,33 & 56 & 10,65 \\
\hline Ninfas & 39 & 48,75 & 431 & 96,67 & 470 & 89,35 \\
\hline Total & 80 & 15,21 & 446 & 84,79 & 526 & 100,00 \\
\hline
\end{tabular}

Tabela 4 - Prevalência da infecção chagásica por localidade em 716 pessoas examinadas no Município de Lassance, MG, em 1999.

\begin{tabular}{lcrc}
\hline & Examinados & \multicolumn{2}{c}{ Positivos } \\
\cline { 3 - 4 } Localidade & $\mathrm{n}^{\circ}$ & $\mathrm{n}^{\circ}$ & 0,00 \\
\hline Barreiro Fundo & 47 & 7 & 6,03 \\
Bela Vista & 116 & 3 & 3,33 \\
Brejo & 90 & 3 & 9,09 \\
Capão & 33 & 2 & 5,71 \\
Nova Lassance & 35 & 0 & 0,00 \\
Piedade & 48 & 2 & 3,70 \\
Santa Mônica & 54 & 4 & 12,50 \\
São Francisco & 32 & 10 & 9,26 \\
Outras localidades* & 108 & 31 & 5,51 \\
Subtotal rural & 563 & 8 & 5,23 \\
Sede Municipal & 153 & 39 & 5,45 \\
\hline Total geral & 716 & & .
\end{tabular}

*: Fazenda Taboquinha, Sítio Buritis, Barro Branco, Gameleira, Santa Rita, Gentil, Barreiro

Fundo, Faz. Salteira, Lavado e Porteiras (com menos de 30 soros colhidos por localidade).

os resultados de 10 localidades rurais com menos de 30 amostras coletadas são apresentados em somatória.

Estatisticamente não foi evidenciada diferença significativa da prevalência entre a Sede Municipal e o conjunto de localidades rurais deste inquérito $(p>0,05)$.

Analisando-se por grupo etário, foi confeccionada a Tabela 5:

Numa segunda etapa realizou-se confirmação sorológica dos indivíduos soropositivos detectados no primeiro exame, então não se confirmando os 3 sororreagentes abaixo dos 20 anos de idade, o que reduziu para 5,03\% a prevalência do Município. O xenodiagnóstico resultou negativo nos 14 pacientes com sorologia positiva em que foi praticado.
Morbidade e mortalidade. A grosso modo, os clínicos locais informam genericamente que não vêem casos agudos e registram basicamente a forma indeterminada e casos de cardiopatia crônica entre indivíduos maiores de 40 anos, registrando ainda uma proporção menor de casos clinicamente suspeitos de megaesôfago e megacólon. Em fevereiro de 2.001, a equipe de investigação deslocou-se para Lassance, com a finalidade de devolver os dados à população e examinar os indivíduos soropositivos (compareceram 29), tendo ainda examinado cerca de 9 outras moradores locais que não haviam participado da sorologia anterior e que sabiam ser portadores de infeção chagásica, totalizando 38 chagásicos examinados. Todos tinham origem rural e residiam em 


\begin{tabular}{|c|c|c|c|c|c|c|}
\hline \multirow{3}{*}{$\begin{array}{l}\text { Faixa etária } \\
\text { (anos) }\end{array}$} & \multicolumn{2}{|c|}{ Zona rural } & \multicolumn{2}{|c|}{ Sede Municipal } & \multicolumn{2}{|c|}{ Total } \\
\hline & examinados & prevalência & examinados & prevalência & examinados & prevalência \\
\hline & $n^{\circ}$ & $(\%)$ & $n^{\circ}$ & $(\%)$ & $n^{\circ}$ & $(\%)$ \\
\hline $0-5$ & 62 & 0,00 & 10 & 0,00 & 72 & 0,00 \\
\hline $6-10$ & 114 & 0,88 & 26 & 0,00 & 140 & 0,71 \\
\hline $11-15$ & 106 & 0,94 & 23 & 0,00 & 129 & 0,78 \\
\hline 16-20 & 47 & 2,13 & 8 & 0,00 & 56 & 1,79 \\
\hline $21-25$ & 21 & 4,76 & 7 & 0,00 & 28 & 3,57 \\
\hline $26-30$ & 20 & 5,00 & 8 & 0,00 & 28 & 3,57 \\
\hline $31-35$ & 39 & 10,26 & 9 & 0,00 & 48 & 8,33 \\
\hline $36-40$ & 38 & 13,15 & 17 & 0,00 & 55 & 9,09 \\
\hline $41-45$ & 36 & 11,11 & 13 & 15,38 & 49 & 12,24 \\
\hline $46-50$ & 32 & 21,87 & 4 & 0,00 & 36 & 30,56 \\
\hline $51-60$ & 27 & 11,11 & 20 & 10,00 & 47 & 10,64 \\
\hline$\geq 61$ & 21 & 14,29 & 18 & 22,22 & 39 & 17,95 \\
\hline Total & 563 & 5,51 & 153 & 5,23 & 716 & 5,45 \\
\hline
\end{tabular}

Lassance, sendo originários do Município 32 (84,21\%) deles e os demais da mesma microrregião. Foram 27 mulheres e 11 homens, entre as idades de 23 e 82 anos. A média das idades foi de 55,13 anos e a mediana 51 anos. Quanto à raça, 47,37 dos examinados eram brancos, $28,95 \%$ mulatos e 23,68 negros. A Tabela 6 mostra que, de modo geral, os antecedentes epidemiológicos mais significativos e registrados neste exame revelaram o padrão comum do chagásico das regiões endêmicas de Minas Gerais.

Quanto ao exame clínico e eletrocardiográfico, todos os 38 pacientes examinados encontravam-se na fase crônica da doença de Chagas; todos lúcidos e ativos, nenhum deles apresentando descompensação cardíaca aparente (Tabela 7).

Tabela 6 - Principais antecedentes pessoais e familiares de 38 pacientes chagásicos examinados em Lassance, MG, em 2001.

\begin{tabular}{lccl}
\hline Antecedentes & Número & $\%$ & \multicolumn{1}{c}{ Observações } \\
\hline Moradia atual (alvenaria) & 30 & 78,95 & adobe: 8 (21,05\%) \\
Galinheiro & 22 & 57,89 & principal ecótopo de . sordida na área \\
Cães em casa & 25 & 65,79 & ratos e gambás: $21(55,26 \%)$ \\
Água encanada & 29 & 76,36 & com esgoto: somente $2(5,26 \%)$ \\
Eletricidade & 33 & 86,84 & com rádio: 71,05 e com TV: $65,79 \%$ \\
Analfabetos & 20 & 52,63 & primário: 17 e secundário: 1 \\
Conhecem o triatomíneo & 34 & 89,47 & viram em casa: $20(52,63 \%)$ \\
Já doou sangue & 4 & 10,53 & nenhum receptor de sangue \\
Tomou ou toma "Rochagan"? & 5 & 13,16 & pacientes fora do inquérito inicial \\
Morte súbita na família & 27 & 71,05 & pacientes mais velhos, principalmente \\
Mal de engasgo na família & 14 & 36,84 & não souberam informar: $5(13,16 \%)$ \\
Parente com doença de Chagas & 29 & 76,32 & maioria total de idosos ou já falecidos \\
\hline
\end{tabular}

O eletrocardiograma apresentou-se dentro dos limites normais em $16(42,11 \%)$ dos 38 pacientes e revelou as alterações comuns da doença de Chagas crônica nos $22(57,89 \%)$ restantes. Os resultados eletrocardiográficos encontram-se resumidos na Tabela 8.

Mortalidade por doença de Chagas. De modo geral, vêm sendo registrados em Lassance entre 30 e 40 óbitos anuais, nos últimos 20 anos, o que representa uma taxa de mortalidade geral por volta de 50/10.000 habitantes/ano. Compulsando-se os dados oficiais de 1980 a 1998, construiu-se a Tabela 9, que corresponde à comparação da mortalidade anual por doença de Chagas em Lassance, em Minas Gerais e no Brasil.
Estes dados mostram uma média em torno de 1,8 óbitos por doença de Chagas em Lassance a cada ano, contribuindo no período com cerca de $5,8 \%$ dos óbitos do município. De modo geral, esta é uma proporção relativamente alta, maior que a do estado de Minas Gerais, por sua vez maior que a do Brasil. De modo geral, há uma tendência de redução relativa dos óbitos por doença de Chagas nos últimos anos para o Brasil e para Minas Gerais.

Já pela pesquisa direta em cartório, buscando-se avaliar mais pormenorizadamente o impacto da esquizotripanose entre janeiro de 1996 e dezembro de 2000, apuraram-se os dados registrados por grupo etário e por sexo, confrontando-se a mortalidade por doença de Chagas frente a outros agravos mais 
Tabela 7 - Situação clínico-eletrocardiográfica geral de 38 chagásicos crônicos examinados em Lassance, MG, fevereiro de 2001.

\begin{tabular}{|c|c|c|c|c|c|c|c|c|c|c|}
\hline \multirow{2}{*}{$\begin{array}{l}\text { Grupos etários } \\
\text { e sexo dos } \\
\text { pacientes }\end{array}$} & \multicolumn{2}{|c|}{$\begin{array}{l}\text { Pacientes } \\
\text { e proporção }\end{array}$} & \multicolumn{2}{|c|}{$\begin{array}{l}\text { Assintomáticos com } \\
\text { clínica e ECG normais }\end{array}$} & \multicolumn{2}{|c|}{$\begin{array}{c}\text { Forma } \\
\text { cardíaca }\end{array}$} & \multicolumn{2}{|c|}{$\begin{array}{l}\text { Disfagia } \\
\text { importante }\end{array}$} & \multicolumn{2}{|c|}{$\begin{array}{l}\text { Obstipação } \\
\text { maior de } 7 \text { dias }\end{array}$} \\
\hline & $\mathrm{n}^{\circ}$ & $\%$ & $\mathrm{n}^{\circ}$ & $\%$ & $n^{\circ}$ & $\%$ & $\mathrm{n}^{\circ}$ & $\%$ & $\mathrm{n}^{\circ}$ & $\%$ \\
\hline $21-40 \mathrm{M}$ & 2 & 28,57 & 1 & 50,00 & 1 & 50,00 & 0 & 00,00 & 0 & 0,00 \\
\hline $21-40 \mathrm{~F}$ & 5 & 71,43 & 3 & 60,00 & 2 & 40,00 & 1 & 20,00 & 0 & 0,00 \\
\hline Subtototal & 7 & 100,00 & 4 & 57,14 & 3 & 42,85 & 1 & 14,28 & 0 & 0,00 \\
\hline $41-60 \mathrm{M}$ & 5 & 29,41 & 2 & 40,00 & 3 & 50,00 & 0 & 0,00 & 1 & 20,00 \\
\hline $41-60 \mathrm{~F}$ & 12 & 70,59 & 7 & 58,33 & 4 & 33,33 & 1 & 8,33 & 0 & 0,00 \\
\hline Subtototal & 17 & 100,00 & 9 & 52,94 & 7 & 41,18 & 1 & 5,88 & 1 & 5,88 \\
\hline$>60 \mathrm{M}$ & 4 & 28,57 & 0 & 0,00 & 4 & 100,00 & 1 & 25,00 & 0 & 0,00 \\
\hline$>60 \mathrm{~F}$ & 10 & 71,43 & 1 & 10,00 & 8 & 80,00 & 1 & 10,00 & 1 & 10,00 \\
\hline Subtototal & 14 & 100,00 & 1 & 7,14 & 12 & 85,71 & 2 & 14,28 & 1 & 7,14 \\
\hline Total M & 11 & 28,95 & 3 & 27,27 & 8 & 72,72 & 1 & 9,09 & 1 & 9,09 \\
\hline Total F & 27 & 71,05 & 11 & 40,74 & 14 & 51,85 & 3 & 11,11 & 1 & 3,70 \\
\hline Total geral & 38 & 100,00 & 14 & 36,84 & 22 & 57,89 & 4 & 10,52 & 2 & 5,26 \\
\hline
\end{tabular}

$\mathrm{M}=$ Masculino $\quad \mathrm{F}=$ Feminino

Tabela 8 - Resultados eletrocardiográficos em 38 pacientes chagásicos de Lassance, MG, por grupo de idade, em 2001.

\begin{tabular}{|c|c|c|c|c|c|c|c|c|}
\hline \multirow[b]{3}{*}{ Resultados } & \multicolumn{6}{|c|}{ Grupos de Idade e $n^{\circ}$ de examinados } & & \\
\hline & \multicolumn{2}{|c|}{$\begin{array}{c}21-40 \\
\text { (7 examinados) }\end{array}$} & \multicolumn{2}{|c|}{$\begin{array}{c}41-60 \\
\text { (17 examinados) }\end{array}$} & \multicolumn{2}{|c|}{$\begin{array}{c}>60 \\
(14 \text { examinados })\end{array}$} & \multicolumn{2}{|c|}{$\begin{array}{c}\text { Total } \\
\text { (38 examinados) }\end{array}$} \\
\hline & $\mathrm{n}^{\circ}$ & $\%$ & $\mathrm{n}^{\circ}$ & $\%$ & $\mathrm{n}^{\circ}$ & $\%$ & $\mathrm{n}^{\circ}$ & $\%$ \\
\hline DLN & 4 & 57,14 & 10 & 58,82 & 2 & 14,28 & 16 & 42,10 \\
\hline ECG alterados & 3 & 42,85 & 7 & 41,18 & 12 & 85,71 & 22 & 57,89 \\
\hline EV Isol & 1 & 14,29 & 1 & 5,88 & 4 & 28,57 & 6 & 17,79 \\
\hline BIRD + HBAE & 1 & 14,29 & 3 & 17,65 & 2 & 14,28 & 6 & 17,79 \\
\hline $\mathrm{BCRD}+\mathrm{HBAE}$ & 2 & 28,57 & 2 & 11,76 & 4 & 28,57 & 8 & 17,79 \\
\hline APT & 2 & 28,57 & 2 & 11,76 & 1 & 7,14 & 5 & 14,29 \\
\hline $\mathrm{BdS}+\mathrm{ASV}$ & - & - & - & - & 1 & 7,14 & 1 & 2,63 \\
\hline EVFP & - & - & 2 & 11,76 & 1 & 7,14 & 3 & 7,89 \\
\hline AIAS & - & - & 3 & 17,65 & 1 & 7,14 & 4 & 10,53 \\
\hline SVE & - & - & - & - & 1 & 7,14 & 1 & 2,63 \\
\hline BV & 1 & 14,29 & - & - & 1 & 7,14 & 2 & 5,26 \\
\hline BCRE & - & - & 1 & 5,88 & - & - & 1 & 2,63 \\
\hline
\end{tabular}

DLN: dentro dos limites normais; EV Isol: extrassístoles ventriculares isoladas; BIRD: bloqueio incompleto de ramo direito; HBAE:hemibloqueio anterior esquerdo;BCRD: bloqueio completo de ramo direito; APT:alteração primária de T;BdS:bradicardia sinusal; ASV: arritmia supraventricular; EVFP: extrassístole ventricular freqüente com polimorfismo;AIAS:área inativa ânteroseptal; SVE: sobrecarga ventricular esquerda; BV: baixa voltagem de QRS; BCRE: bloqueio completo de ramo esquerdo.

Observação: duas ou mais alterações podem estar presentes no mesmo eletrocardiograma.

Tabela 9 - Mortalidade anual por doença de Chagas registrada em Lassance, em Minas Gerais e no Brasil, entre 1980 e 1998.

\begin{tabular}{|c|c|c|c|c|c|c|}
\hline \multirow[b]{2}{*}{ Ano } & \multicolumn{2}{|c|}{ Lassance } & \multicolumn{2}{|c|}{ Minas Gerais } & \multicolumn{2}{|l|}{ Brasil } \\
\hline & $\begin{array}{c}\text { Mortes por } \\
\text { doença de Chagas }\end{array}$ & $\begin{array}{l}\text { Mortes por d. Chagas } \\
\text { total de mortes (\%) }\end{array}$ & $\begin{array}{c}\text { Mortes por } \\
\text { doença de Chagas }\end{array}$ & $\begin{array}{r}\text { Mortes por d. Chagas } \\
\text { total de mortes (\%) }\end{array}$ & $\begin{array}{c}\text { Mortes por } \\
\text { doença de Chagas }\end{array}$ & $\begin{array}{c}\text { Mortes por d. de Chagas } \\
\text { total de mortes (\%) }\end{array}$ \\
\hline 1980 & 1 & 4,76 & 2.227 & 2,35 & 6.191 & 0,82 \\
\hline 1982 & 2 & 5,41 & 2.182 & 2,41 & 6.139 & 0,83 \\
\hline 1984 & 4 & 12,12 & 2.101 & 2,28 & 6.274 & 0,77 \\
\hline 1986 & 3 & 8,12 & 2.100 & 2,24 & 6.159 & 0,76 \\
\hline 1988 & 0 & 0,00 & 2.088 & 2,23 & 6.257 & 0,75 \\
\hline 1990 & 2 & 9,09 & 1.937 & 2,14 & 5.845 & 0,72 \\
\hline 1991 & 1 & 4,35 & 1.859 & 2,08 & 5.529 & 0,69 \\
\hline 1992 & 3 & 9,09 & 1.800 & 1,90 & 5.710 & 0,69 \\
\hline 1993 & 1 & 2,44 & 1.800 & 1,83 & 5.772 & 0,66 \\
\hline 1994 & 1 & 5,26 & 1.695 & 1,74 & 5.549 & 0,63 \\
\hline 1995 & 1 & 3,45 & 1.590 & 1,66 & 5.442 & 0,61 \\
\hline 1996 & 3 & 8,47 & 1.509 & 1,56 & 5.376 & 0,59 \\
\hline 1997 & 3 & 9,37 & 1.667 & 1,70 & 5.410 & 0,60 \\
\hline
\end{tabular}

Fonte: Ministério da Saúde (DATASUS) 
significativos, anotando-se ainda os óbitos registrados sem assistência médica. Nestes dados, mostra-se a importância relativa da doença de Chagas como causa de óbitos entre adultos, especialmente a partir

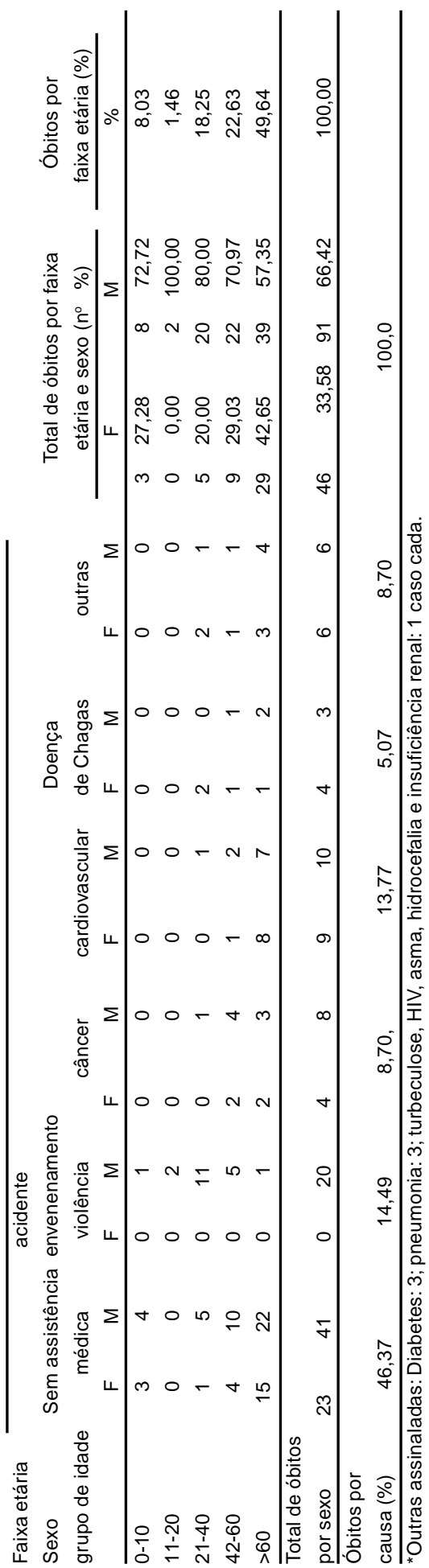

da $4^{a}$ década de vida, podendo esta proporção ser maior, frente à vultosa incidência de óbitos sem assistência médica e mesmo aqueles tachados como de causa cardiovascular (Tabela 10).

\section{DISCUSSÃO E CONCLUSÕES}

Do ponto de vista histórico, Lassance apresenta uma evolução similar à de sua região, pontificando-se seu crescimento no contexto da chegada da ferrovia, que foi de definitiva importância para viabilizar o desenvolvimento regional, a partir de 1910. Parte deste, sem dúvida, também poderá creditar-se a Carlos Chagas, à raiz do excelente trabalho por ele desenvolvido, contra o paludismo. Os surtos de progresso e urbanização se seguiram em Lassance, alternados com períodos de depressão e expulsão populacional, registrados em toda a região ${ }^{122030}$. Lassance chegou a Município em 1953 e tem se esforçado por modernizar-se e tornar-se economicamente sustentável, o que é difícil para pequenas populações em regiões mais isoladas e de terras empobrecidas ${ }^{101320}$. Mesmo melhorando nos últimos anos seu acesso e meios de comunicação, através do aporte de rodovia pavimentada, telefonia e televisão, Lassance segue pequena, com população rural proporcionalmente decrescente, uma regra geral para a região. Não obstante os recentes baques econômicos, a população segue buscando outras formas de ativação econômica, principalmente a partir de maior produção no setor primário (agricultura e pecuária) e, mesmo, o turismo $\mathrm{m}^{30}{ }^{37}$. Isto poderá proporcionar uma lento crescimento demográfico, particularmente da área urbana $^{1320}$. Neste contexto, estando o crescimento econômico basicamente dependente de modernização rural, é provável que a doença de Chagas dificilmente voltará a ser o que já foi neste Município ${ }^{20} 33$

A descoberta de Chagas foi num momento de implantação de focos domiciliares de $P$. megistus em Lassance, provavelmente à custa de insetos originários de regiões mais meridionais e carreados passivamente por movimentos populacionais e pelo próprio avanço da ferrovia, como registrado na Bahia, em Minas e em São Paulo5 12102935.

Provavelmente, houve transmissão da doença humana em Lassance, antes de Chagas, através de focos esparsos de triatomíneos de outras espécies naturalmente existentes na região, a julgar pelo encontro de reservatórios silvestres infectados em Lassance, e por observações entomológicas na área, nos primeiros $\operatorname{anos}^{5} 722$. O triatomismo domiciliar de Lassance, no entanto, fica basicamente por conta de P. megistus (1908 a 1960) e de T. infestans (1950-1960 a 1980), principais causadores, ali, da infeção do homem ${ }^{5171928}$. Sem dúvida, Lassance foi um foco importante de transmissão e expansão da doença de Chagas humana, principalmente até os anos 80 , o que se nota pelos dados disponíveis de prevalência no Município e Região e, até hoje, pelos dados de mortalidade, em proporções superiores ao do estado e do Brasil ${ }^{17}$. Foi marcante a influência do controle químico dos vetores no município, 
a partir de 1983, resultando em virtual eliminação de $T$. infestans e $P$. megistus no município, o que explica a interrupção da transmissão da infeção às novas gerações de habitantes.

Verifica-se também que a moléstia tem sido importante causa de morbi-mortalidade e danos sociais em Lassance desde a descoberta, a julgar pelos dados de mortalidade colocados por Chagas e, mais tarde também de aposentadoria na área ${ }^{617}$. Ressaltam, em particular, as referências dos 38 pacientes presentemente examinados, citando altos percentuais de chagásicos e morte súbita em seus antepassados, assim como 36,84\% de mal de engasgo, que são marcadores muito sugestivos da endemia chagásica em outras áreas brasileiras $^{12} 15{ }^{35}$. Os dados atuais mostram uma infestação de média-baixa intensidade por T. sordida em Lassance, basicamente no peridomicílio e com a ausência de insetos infectados pelo T. cruzi. Isto se reflete na ausência da infeção humana em baixas idades, na prática significando eliminação da transmissão vetorial aliás, como ocorrido em São Paulo ${ }^{27} 33$.

Como panorama atual, fica em Lassance um resíduo de indivíduos mais idosos ainda portadores da doença de Chagas, com a qual se infectaram há duas décadas ou mais.a merecer atenção médica e social. São pessoas de origem rural, geralmente pobres e de baixa escolaridade, tudo indicando como vetorial sua provável fonte de infecção, como de regra se observa nos municípios mineiros ${ }^{12}{ }^{15}$. Chama a atenção, a ausência de xenodiagnósticos positivos, o que sói acontecer em populações idosas de áreas sem transmissão ativa da doença ${ }^{12}$. No plano clínico, estes indivíduos em geral estão bem, compensados, neles desenvolvendo-se as formas mais comuns da cardiopatia crônica chagásica, assim como, provavelmente e em menor proporção, as formas digestivas habituais. Nota-se que as formas clínicas mais comuns da doença crônica ocorrem nas proporções usuais para as áreas endêmicas de Minas, ressaltando uma proporção relativamente alta de assintomáticos sem forma cardíaca mesmo em idades entre 40 e 60 anos, com forte tendência de queda a partir dos 60 (Tabela 7) ${ }^{15}$. A detecção de 22,86\% de casos de BCRD + HBAE é fortemente sugestiva de cardiopatia chagásica, nas áreas endêmicas ${ }^{15}$.

Por outro lado, em Lassance remanesce uma população triatomínica nativa e de baixa intensidade, praticamente não infectada pelo parasito, com muito pouco potencial de domiciliação e de transmissão do T.cruzi ao ser humano. Este ponto faz pressupor o funcionamento de um esquema perene e eficaz de vigilância epidemiológica, o que já foi estabelecido no município e reforçado com a realização do presente trabalho ${ }^{15} 19$.

Como conclusão geral, presentemente Lassance se encontra livre da transmissão da doença de Chagas, ali tão importante do ponto de vista histórico e epidemiológico. Os indivíduos infectados são poucos, de grupos etários mais elevados, ocorrendo ainda mortalidade relativamente expressiva pela doença. Em particular, a pequenina Lassance pode e deve viver, hoje, a memória bonita dos feitos de Carlos Chagas, ao mesmo tempo que luta para se viabilizar sócioeconomicamente, devendo ainda permanecer atenta e atuante para que a doença não volte a se implantar entre os seus habitantes.

\section{REFERÊNCIAS BIBLIOGRÁFICAS}

1. Akhavan D. Análise de custo-efetividade do programa de controle da doença de Chagas no Brasil. Relatório final. Organização Panamericana da Saúde, Brasília, 2000.

2. Cançado JR. Forma aguda da doença de Chagas no Brasil. In: Almeida AO (ed) Modernos conhecimentos sobre doença de Chagas. Academia Mineira de Medicina, Belo Horizonte, p. 13-28, 1985.

3. Chagas CRJ. Moléstia de Carlos Chagas. Segunda Conferência realizada na Academia Nacional de Medicina, em agosto de 1911. Tipografia Leuzinger, Rio de Janeiro, 1911.

4. Chagas CRJ. Tripanosomiase Americana. Forma aguda da moléstia. Memórias do Instituto Oswaldo Cruz 8: 37-69, 1916.

5. Chagas CRJ. Trypanosomiase americana. Sinonimia: doença do barbeiro. Revista do Brasil 32: 1-33, 1918.

6. Chagas CRJ. Estado actual da Tripanosomiase americana. Revista de Biologia e Hygiene 5: 58-64, 1934.

7. Chagas CRJ. Lassance, 1907. In: Cançado JR, Chuster M (orgs.) Cardiopatia Chagásica. Fundação Carlos Chagas, Belo Horizonte, p. 391-413, 1984.

8. Chagas Filho C. Histórico sobre a doença de Chagas. In: Cançado JR (ed) Doença de Chagas, Imprensa Oficial, Belo Horizonte, p. 5-21, 1968.

9. Chagas Filho C. Meu Pai. Editora Fundação Oswaldo Cruz, Rio de Janeiro, 1993.
10. Dias E. Um ensaio de profilaxia de moléstia de Chagas. Rio de Janeiro, Imprensa Oficial, 1945.

11. Dias E, Serebrenick S. Aspectos climáticos e distribuição dos transmissores da doença de Chagas no Vale do Rio São Francisco. Memórias do Instituto Oswaldo Cruz 56: 407-416, 1958.

12. Dias JCP. Doença de Chagas em Bambuí, Minas Gerais, Brasil. Estudo clínico-epidemiológico a partir da fase aguda, entre 1940 e 1982. Tese de Doutorado Universidade Federal de Minas Gerais, Belo Horizonte, 1982.

13. Dias JCP. Rural resource development and its potential to introduce domestic vectors into new epidemiological situation. Revista Argentina de Microbiología 20: 81-85, 1988

14. Dias JCP. Atualidade de Carlos Chagas. In: Casa de Oswaldo Cruz: Biblioteca Virtual Carlos Chagas.: http://www.prossiga.br/ chagas/, 1999.

15. Dias JCP, Coura JR. Epidemiologia. In : Dias JCP, Coura JR (orgs.) Clínica e Terapêutica da doença de Chagas. Uma abordagem prática para o clínico geral. Editora Fundação Oswaldo Cruz, Rio de Janeiro, p. 33-66, 1997.

16. Dias JCP, Loyola CCP, Brener S. Doença de Chagas em Minas Gerais: situação atual e perspectivas. Revista Brasileira de Malariologia e Doenças Tropicais 37: 7-28, 1985.

17. Dias JCP, Schofield CJ. The Evolution of Chagas Disease (American Trypanosomiasis) Control after 90 years since Carlos 
Chagas Discovery. Memórias do Instituto Oswaldo Cruz 94 (supl I): 103-121, 1999.

18. Fundação João Pinheiro. Formação Histórica da Região Noroeste de Minas Gerais (1690-1967). Monografia. Belo Horizonte, 1999.

19. Fundação Nacional de Saúde. Normas Técnicas para o Controle da Doença de Chagas. Ministério da Saúde, Brasília, 1996.

20. Fundação Nacional de Saúde-MG. Registros e Arquivos disponíveis. Rua Espírito Santo 500/9 andar, Belo Horizonte, MG, 1999.

21. Lent $H$, Wigodzinsky P. Revision of the triatominae (Hemiptera, Reduviidae), and their significance as vectors of Chagas' disease. Bulletin of American Museum of Natural History (New York) 163: 123-520, 1979.

22. Lutz A, Machado A. Viagem pelo Rio São Francisco e por alguns de seus afluentes, entre Pirapora e Juazeiro. Memórias do Instituto Oswaldo Cruz 7: 5-62, 1915.

23. Martins AV, Versiani V, Tupinambá A. Estudos sobre a tripanosomíase americana em Minas Gerais, Brasil. Memórias do Instituto Oswaldo Cruz 35: 285-301, 1940.

24. Mello BH. Atlas do Brasil. Briguiet Editora, Rio de Janeiro, 1909.

25. Neiva A. Informações sobre a biologia da vinchuca, Triatoma infestans Klug. Memórias do Instituto Oswaldo Cruz 5:24-31, 1913.

26. Neiva A, Penna B. Viagem científica pelo norte da Bahia, sudoeste de Pernambuco, sul do Pauí e norte e sul de Goiás. Memórias do Instituto Oswaldo Cruz VIII: 3-133, 1916.

27. Organização Panamericana da Saúde. Informe de La Mission de Evaluación de las Actividades de Control/Eliminación de T. infestans y Corte de la Transmisión Transfusional de Chagas: Estados de São Paulo y Minas Gerais. Washington, Documento Interno, 1955.
28. Pellegrino J. Novos dados sobre a distribuição de triatomíneos e sua infeção pelo Schizotrypanum cruzi no Estado de Minas Gerais, Brasil. Memórias do Instituto Oswaldo Cruz 48: 639-667, 1950.

29. Pirajá da Silva MA. O barbeiro (Conorrhinus megistus Burm.) na Bahia. Arquivos da Sociedade Brasileira de Medicina 1: 627-632, 1913.

30. Prefeitura Municipal de Lassance. Lassance. Histórico do Município. Mimeo, 1999.

31. Salgado AA, Pellegrino J. Distribuição Geográfica. In: Cançado JR (ed) Doença de Chagas. Imprensa Oficial, Belo Horizonte p.143-162, 1968.

32. Schenone $H$, Alfaro E, Rojas A. Bases y rendimiento del xenodiagnóstico en la infección chagásica humana. Boletín Chileno de Parasitología 29: 24-26, 1974.

33. Schofield CJ, Dias JCP. The Southern Cone Initiative against Chagas Disease. Advances in Parasitology 42: 2-30, 1998.

34. Sherlock I. Vetores. In:Brener Z, Andrade ZA (orgs) Trypanosoma cruzi e Doença de Chagas. Editora Guanabara Koogan, Rio de Janeiro, p. 42-88, 1979.

35. Silva LJ. Evolução da doença de Chagas no Estado de São Paulo. Tese, Faculdade de Medicina da Universidade de São Paulo, Ribeirão Preto, 1980.

36. Silveira AC, Feitosa V, Borges R. Distribuição de triatomíneos capturados no ambiente domiciliar, no período 1975-83, Brasil. Revista Brasileira de Malariologia e Doenças Tropicais 36: 15-312, 1983.

37. Vieira Neto M. Lassance: o berço de Carlos Chagas. Monografia. Prefeitura Municipal, Lassance, 1999.

38. Villela E. Coletânea de Textos sobre Carlos Chagas. Instituto Oswaldo Cruz, Rio de Janeiro, 1959. 